This is a post-peer-review, pre-copy edited version of an article published in Sozialer

Fortschritt. The definitive publisher-authenticated version (https://doi.org/10.3790/sfo.67.8-

9.667) is available online at: https://elibrary.duncker-

humblot.com/journals/id/21/vol/67/iss/1784/art/8875/

\title{
Wer hat Zugang zu Elterngeld? \\ Soziale Rechte und Anspruchsbedingungen in vergleichender Perspektive ${ }^{1}$
}

\begin{abstract}
Die vergleichende Forschung hat sich bislang mit den Anspruchsbedingungen für Elterngeldleistungen, v.a. aber mit den Rechtsansprüchen von Eltern mit geringerer Arbeitsmarktbindung, wenig auseinandergesetzt. Hierdurch blieben soziale Ungleichheiten beim Zugang zu Elterngeld weitgehend unerforscht. Sie sind insbesondere in denjenigen Ländern von Bedeutung, in denen Elterngeldansprüche an eine bestimmte Form oder Dauer des Beschäftigungsverhältnisses geknüpft sind. Aufbauend auf der Literatur zu sozialen Rechten entwickeln wir in diesem Beitrag einen konzeptionellen Rahmen, der erfasst, inwiefern Elterngeldansprüche (un-)abhängig von der Arbeitsmarktposition der Eltern gewährt werden. Auf dieser Basis unterscheiden wir vier Idealtypen: das universale Elternschafts-Modell, das selektive Elternschafts-Modell, das universale Adult-Worker-Modell sowie das selektive Adult-Worker-Modell. Abschließend illustrieren wir diese Typen mit Beispielen von Elterngeldregelungen in drei verschiedenen Ländern: Deutschland, Belgien und Kroatien.
\end{abstract}

\begin{abstract}
There is limited knowledge about eligibility for leave benefits in general, and about leave rights of parents less securely attached to the labour market in particular. Consequently, social inequalities in access to leave benefit rights remain hidden, which may be particularly pronounced in countries where a certain duration or form of employment is a principal condition to exercise leave rights. In this chapter, we develop an innovative conceptual framework based on the social rights literature, which takes into account how access to parental leave benefits is granted (in-)dependent of labour market position. Four ideal types are presented: the universal parenthood model, the selective parenthood model, the universal adult-worker model, and the selective adult-worker model. Finally, we illustrate these types with three country case of parental leave systems: Germany, Belgium, and Croatia.
\end{abstract}

\footnotetext{
${ }^{1}$ Dieser Beitrag fußt zu weiten Teilen auf einer Übersetzung unseres Beitrags im Buch „Parental Leave and Beyond“ (Hrsg. von Ann-Zofie Duvander, Alison Koslowski und Peter Moss, erscheint 2019). Wir bedanken uns bei Policy Press für die Genehmigung zum übersetzten Wiederabdruck dieses Beitrags. Für wertvolle Hinweise zur Überarbeitung des Beitrags danken wir außerdem den Herausgeberinnen des Sonderhefts, Diana Auth, Florian Blank und Michaela Schulze, sowie den beiden Gutachtern.
} 


\section{Einleitung}

Es bestehen große Länderunterschiede dahingehend, inwiefern Elterngeldleistungen ${ }^{2}$ (un-)abhängig von der Arbeitsmarktposition der Eltern gewährt werden. Dies ist äußerst relevant im Hinblick auf die möglichen Wirkungen unterschiedlicher Regelungen - insbesondere sozialer Ungleichheiten im Zugang zu Elterngeld. So scheinen z.B. Vereinbarkeitsmaßnahmen insbesondere denjenigen Eltern zugute zu kommen, die bereits am Arbeitsmarkt partizipieren, vor allem Familien mit zwei berufstätigen Elternteilen und mit höherem Einkommen (Cantillon 2011; Ghysels/van Lancker 2011). Es kann daher davon ausgegangen werden, dass Ungleichheiten auf dem Arbeitsmarkt auch für Elterngeldrechte zentral sind. Dennoch hat sich die vergleichende Literatur in erster Linie auf Elterngeldansprüche für erwerbstätige Eltern konzentriert. Im Fokus stehen in der Regel die Länge der Elternzeit sowie die Höhe der Elterngeldansprüche (in geringerem Maße auch Fragen nach der Übertragbarkeit und Flexibilität der Ansprüche), sowie die Auswirkungen verschiedener Regelungen auf Erwerbstätigkeit und geschlechtliche Arbeitsteilung (für einen Literaturüberblick siehe z.B. Dobrotić 2015; McKay et al. 2016). Demgegenüber wissen wir nur wenig über den Zugang zu Elterngeldleistungen, v.a. entsprechend der Erwerbshistorie der Eltern (Javornik 2014).

Um den vorherrschenden Fokus auf Elterngeldleistungen mit der - vorgelagerten - Frage nach dem Zugang zu diesen Leistungen zu verknüpfen, bietet es sich an, auf der Literatur zu sozialen Bürgerrechten aufzubauen, die diese unterschiedlichen Dimensionen abbildet (Clasen/Clegg 2007; Blank 2011). In einigen Ländern hängt der Zugang zu Elterngeldrechten in hohem Maße von der (Form der) Arbeitsmarktpartizipation ab. In Belgien z.B. besteht nur dann ein Anspruch, wenn zuvor eine vergleichsweise lange, ununterbrochene Beschäftigung bei derselben Arbeitgeberin ${ }^{3}$ bestanden hat (Merla 2017). In anderen Ländern, darunter Deutschland, existiert zumindest auf eine BasisElterngeldleistung ein universaler Anspruch, d.h. für alle Eltern, unabhängig von einer vorherigen Erwerbstätigkeit oder deren Form (Reimer et al. 2017). Wenn der Anspruch auf Elterngeld an bestimmte Voraussetzungen des Beschäftigungsverhältnisses geknüpft ist (z.B. nur stabile Beschäftigung oder nur abhängige Beschäftigung zu einer Anspruchsberechtigung führt), so kann dies soziale Ungleichheiten im Leistungszugang weiter verstärken. Bislang gibt es jedoch wenig Forschung, die sich tiefergehend damit auseinandersetzt, wie die Ausgestaltung von Elterngeldansprüchen mit sozialen Ungleichheiten zusammenhängt (McKay et al. 2016).

In einem ersten Schritt ist es daher wichtig zu verstehen wie Elterngeldansprüche mit der Arbeitsmarktposition von Eltern zusammenhängen; insbesondere mit Blick auf Eltern, die einer atypischen Beschäftigung nachgehen oder nicht am Arbeitsmarkt partizipieren. Dieses Ziel verfolgt der vorliegende Beitrag. Er konzentriert sich, um Typen zu identifizieren, auf Elterngeldleistungen, d.h. Geldleistungen, die Müttern und Vätern nach den anfänglichen Mutterschutz- bzw.

\footnotetext{
${ }^{2}$ In der internationalen Forschung werden unter parental leave sowohl Zeit- als auch Geldleistungen gefasst; im deutschen Sprachgebrauch wird beides voneinander abgegrenzt (Elternzeit bzw. Elterngeld). Dies ist auch insofern klarer, als dass - in Deutschland, aber auch in einigen anderen Ländern - die gesetzlichen Grundlagen unterschiedlich sein können und die Leistungen häufig nicht direkt zusammenhängen, z.B. ist nicht überall für den Elterngeldbezug auch die Inanspruchnahme von Elternzeit erforderlich, in anderen Ländern wiederum besteht nur eine unbezahlte Elternzeit (Blum et al. 2017). .

${ }^{3}$ Wir verwenden in losem Wechsel die weibliche und männliche Personenform. Personen des jeweils anderen Geschlechts sind an diesen Stellen mit gemeint.
} 
Väterzeitleistungen ${ }^{4}$ zur Verfügung stehen. Elterngeldansprüche sind am besten geeignet, um die Rechte von erwerbslosen Eltern oder Eltern mit atypischem Beschäftigungsverhältnis abzubilden. Während nämlich Mutterschutz- und Väterzeitleistungen i.d.R. auf erwerbstätige Eltern beschränkt sind (siehe z.B. Blum et al. 2017), haben Elterngeldleistungen häufig einen breiteren Kreis an Anspruchsberechtigten, der auch inaktive und arbeitslose Personen einschließt. Zudem möchten wir uns auf die monetäre Leistung konzentrieren, da diese in Hinblick auf soziale Ungleichheiten aussagekräftiger ist als der Anspruch auf Elternzeit, der typischerweise an weniger Bedingungen geknüpft und als arbeitsrechtlicher (Kündigungs-)Schutz konzipiert ist. ${ }^{5}$ Auf Grundlage der Literatur zu sozialen Bürgerrechten entwickeln wir einen konzeptionellen Rahmen, der unterscheidet, inwiefern Rechtsansprüche auf Elterngeld (un-)abhängig von der (Form der) Arbeitsmarktposition der Eltern bestehen. Insgesamt können vier Idealtypen identifiziert werden. Ausgeblendet bleibt an dieser Stelle, ob Elterngeldrechte für verschiedene Familientypen gelten (z.B. Alleinerziehende, gleichgeschlechtliche Familien). Abschließend werden wir die identifizierten Idealtypen mit Beispielen von Elterngeldregelungen aus drei verschiedenen Ländern veranschaulichen (Deutschland, Belgien und Kroatien).

\section{Konzeptioneller Rahmen: Soziale Rechte auf Elterngeld}

Ähnlich wie auf anderen sozialpolitischen Feldern, hängen auch die Unterschiede bei Elterngeldregelungen mit den jeweiligen ideologischen Vorstellungen von sozialer Bürgerschaft zusammen (Kvist 2005). Zentral ist dabei die Rolle des Staates und in welchem Maße er Individuen mit Möglichkeiten und Ressourcen ausstatten soll (Clasen/Clegg 2007). Je nachdem, welche Vorstellung von sozialer Bürgerschaft in einem bestimmten Zeitraum oder Land vorherrscht, können Elterngeldregelungen verschiedene soziale Gruppen bevorzugen bzw. benachteiligen. Ebenso können Männer und Frauen unterschiedlich behandelt werden; soziale Bürgerschaft ist keineswegs geschlechtsneutral (Orloff 1993). Vielmehr hängen die bestehenden Policies in hohem Maße mit sich ändernden Vorstellungen über die Aufteilung von bezahlter und unbezahlter Arbeit zusammen (Kurowska 2016). Auch Gender-Ungleichheiten der Elterngeldansprüche werden daher im Folgenden angrenzend beleuchtet; der Fokus liegt aber auf den sozialen Ungleichheiten.

\subsection{Drei Dimensionen sozialer Rechte}

Ein geeigneter Ausgangspunkt zur Untersuchung sozialer Rechte sind die Arbeiten von Marshall (1964), der soziale Rechte als letzte Stufe der Entwicklung des Bürgerstatus ansieht, nach den zivilen und den politischen Rechten. Typischerweise haben soziale Rechte einen ökonomischen Charakter, da sie einen Anspruch auf monetäre Transfers, Güter und Leistungen nach sich ziehen und in

\footnotetext{
${ }^{4}$ In Deutschland existiert keine spezielle „Väterzeit“, in vielen anderen Ländern (z.B. Belgien, Portugal) ist dies aber der Fall (Blum et al. 2017). Väterzeitregelungen begründen i.d.R. einen Anspruch auf einige Tage bis Wochen Arbeitsfreistellung, der zeitnah nach der Geburt eines Kindes eingelöst wird. In Deutschland kann in diesem Zeitraum, parallel zum Mutterschutz, vom Vater Elternzeit genommen werden, was wiederum in einigen anderen Ländern (z.B. Österreich) nicht möglich ist.

${ }^{5}$ So haben einige Länder nur eine unbezahlte Elternzeit (z.B. Griechenland, Irland, Spanien, Großbritannien), auch wenn dann z.T. regionale Leistungen existieren (Spanien) oder es Unterschiede zwischen öffentlichem Dienst und der Privatwirtschaft gibt (Griechenland) (Blum et al. 2017).
} 
Wechselwirkung mit dem Arbeitsmarkt definiert werden (Blank 2011). Zentrale Überlegungen zu sozialen Rechten hat Esping-Andersen (1990) mit seinem Konzept der De-Kommodifizierung vorgenommen: „If social rights are given the legal and practical status of property rights, if they are inviolable, and if they are granted on the basis of citizenship rather than performance, they will entail a de-commodification of the status of individuals vis-à-vis the market" (Esping-Andersen 1990, S. 21). Auch wenn soziale Rechte nicht notwendigerweise de-kommodifizierend angelegt sind (Blank 2011), so ist der Grad der Unabhängigkeit von kapitalistischen Arbeitsmärkten (z.B. im Fall von Alter, Arbeitslosigkeit oder eben Elternschaft) doch konstitutiv.

Soziale Rechte können an Erwerbsarbeit geknüpft sein (z.B. durch Sozialversicherungen), aber auch an den Bürgerstatus oder an „Bedürftigkeit“" (Esping-Andersen 1990). „Bürgerstatus“ als Basis sozialer Rechte wird hier als Sammelbegriff verwendet, denn de facto sind hier häufig auch (äquivalente) Rechte eingeschlossen, die im Fall eines (langfristigen) Wohnsitzes im Land bestehen. Für Asylsuchende und Flüchtlinge gelten z.T. abweichende Regelungen zu anderen Migranten, allerdings bestehen hier deutliche Unterschiede zwischen den EU-Mitgliedsstaaten (Europäisches Parlament 2015). Innerhalb der EU ist die Situation auch insofern eine besondere, als dass EU-Bürger durch Erwerbstätigkeit in einem anderen Mitgliedsstaat (unter bestimmten Bedingungen auch deren Familien) einen zu den Staatsbürgern dieses Landes gleichgestellten Zugang zu Sozialleistungen erhalten können.

Diese unterschiedlichen Grundlagen bilden die erste von insgesamt drei Dimensionen sozialer Rechte (Blank 2007, 2011; Clasen/Clegg 2007), nämlich das (1) Anspruchsprinzip. Wohlfahrtsstaaten greifen in sehr unterschiedlicher Weise auf verschiedene Anspruchsprinzipien zurück, um soziale Rechte zu gewähren. Nimmt man wiederum auf Esping-Andersen (1990) Bezug, so tendieren sozialdemokratische Wohlfahrtsstaaten dazu, soziale Rechte auf der Basis von Bürgerschaft einzurichten. Konservative Wohlfahrtsstaaten nutzen v.a. erwerbszentrierte Sozialversicherungen. In liberalen Wohlfahrtsstaaten hingegen ist die „Bedürftigkeit“ das dominierende Anspruchsprinzip; sie wird durch Bedarfsprüfungen oder niedrige Pauschalleistungen identifiziert. Zudem werden bei der Gewährung sozialer Rechte typischerweise verschiedene Anspruchsprinzipien kombiniert - vor allem in der Familienpolitik. Wie Sainsbury (1996, S. 22) konstatiert, werden Ansprüche nicht notwendigerweise durch Bürgerschaft oder Erwerbstätigkeit begründet, sondern teils auch durch Elternschaft oder die Ehe. Im Unterschied zum Konzept der De-Kommodifizierung müssen hier also auch die sozialen Rechte der ökonomisch Abhängigen (zumeist Frauen) erfasst werden (Lewis 2001; Orloff 1993). Ein typisches Beispiel ist die Kombination von sozialversicherungs- und ehebasierten Rechten in konservativen Wohlfahrtsstaaten: Die auf Sozialversicherung basierenden Leistungen sind dabei auf die (traditionell: männlichen) Erwerbstätigen und auf die Absicherung „alter sozialer Risiken" (Bonoli 2005) ausgelegt, während die auf Ehe basierenden Rechte auf die (traditionell: weiblichen) abhängigen Familienangehörigen ausgerichtet sind, um deren Risiko einer fehlenden Arbeitsmarktpartizipation zu reduzieren. Individuelle Rechte, die auf Erwerbstätigkeit basieren, sind eher für de-familialisierende Regime charakteristisch, auf Ehe basierende soziale Rechte hingegen für familialisierende Regime. Dieser geschlechtsspezifische Charakter sozialer Rechte ist bei Elterngeldpolitiken deshalb von hervorgehobener Bedeutung, da ihre Ausgestaltung die geschlechtsspezifischen Ungleichheiten im Haushalt und auf dem Arbeitsmarkt stark beeinflusst (z.B. Dearing 2016). 
Die zweite Dimension sozialer Rechte bezieht sich auf die Programmebene. Hier ist es entscheidend, auch die (2) Anspruchskriterien einzubeziehen, die den sozialen Rechten vorgeschaltet sind (Clasen/Clegg 2007). Der klassische Fokus auf sozialen Rechten, die auf Erwerbstätigkeit basieren (und De-Kommodifizierung als einer der Hauptindikatoren), kann mit Blick auf soziale Ungleichheiten kritisch diskutiert werden. Leistungen mit Einkommensersatzfunktion, die auf regulär Beschäftigte abzielen, kommen v.a. mittleren und höheren Einkommensgruppen zugute und können soziale Ungleichheiten verstärken. Dies kann u.a. soziale Ungleichheiten zwischen verschiedenen Gruppen am Arbeitsmarkt betreffen, z.B. zwischen Arbeitnehmern mit befristeter versus unbefristeter Anstellung (Blofield/Martinez Franzoni 2015). Neben den geschlechtsspezifischen Wirkungen sozialer Sicherungssysteme ist daher auch entscheidend, ob Policies bestehende Arbeitsmarktunterschiede eher abschwächen oder verstärken. So stellt sich z.B. die Frage, inwiefern durch eine bestimmte Regelung alle auf dem Arbeitsmarkt aktiven Personen abgesichert werden oder nur bestimmte Gruppen (indem z.B. Selbstständige oder atypisch Beschäftigte ausgeschlossen bleiben). Über Bedarfsprüfungen können soziale Rechte überdies von der individuellen Bedürftigkeit abhängig sein. Die zweite Dimension sozialer Rechte erlaubt also Rückschlüsse auf soziale Ungleichheiten.

Die dritte Dimension sozialer Rechte bezieht sich auf ihren tatsächlichen Inhalt, d.h. (3) den Leistungsumfang. Wie bereits Marshall (1950, in Orloff 1993, S. 28-29) konstatiert hat, existiert kein universales Prinzip, das über die Verteilung von Rechten und Pflichten bestimmen würde. Vielmehr können verschiedene Anspruchsprinzipien mit unterschiedlichen Leistungshöhen einhergehen. So hat Orloff (1993) hervorgehoben, dass erwerbsbasierte Rechte normalerweise generöser sind als universale Rechte oder abgeleitete Rechte, die auf dem Ehestatus basieren (und die zudem mit strengeren Anspruchskriterien einhergehen). Nichtsdestotrotz: Die vergleichende Sozialpolitikforschung hat sich häufig auf die dritte Dimension des Leistungsumfangs konzentriert, u.a., da sie am einfachsten quantifizierbar ist (z.B. Leistungsdauer und -höhe). Demgegenüber existiert nur begrenztes Wissen über die „Auflagenseite“ (Clasen/Clegg 2007; Blank 2007) der sozialen Bürgerschaft, d.h. die Voraussetzungen, die über Zugang oder Ausschuss von Leistungen sowie deren Umfang bestimmen. Diese Feststellung trifft auf die Forschung zu Elternzeit und Elterngeld in besonderem Maße zu; hier sind gesetzliche Ansprüche und Anspruchskriterien relativ unerforscht (Javornik 2014). Vor diesem Hintergrund zeigt ein dreidimensionaler Blick auf soziale Rechte (Abb. 1), welche Formen und welches Ausmaß an Unterstützung unter welchen Bedingungen in Anspruch genommen werden kann (Blank 2007, S. 8). 


\section{Abb. 1: Soziale Rechte - Dreidimensionaler konzeptioneller Rahmen}

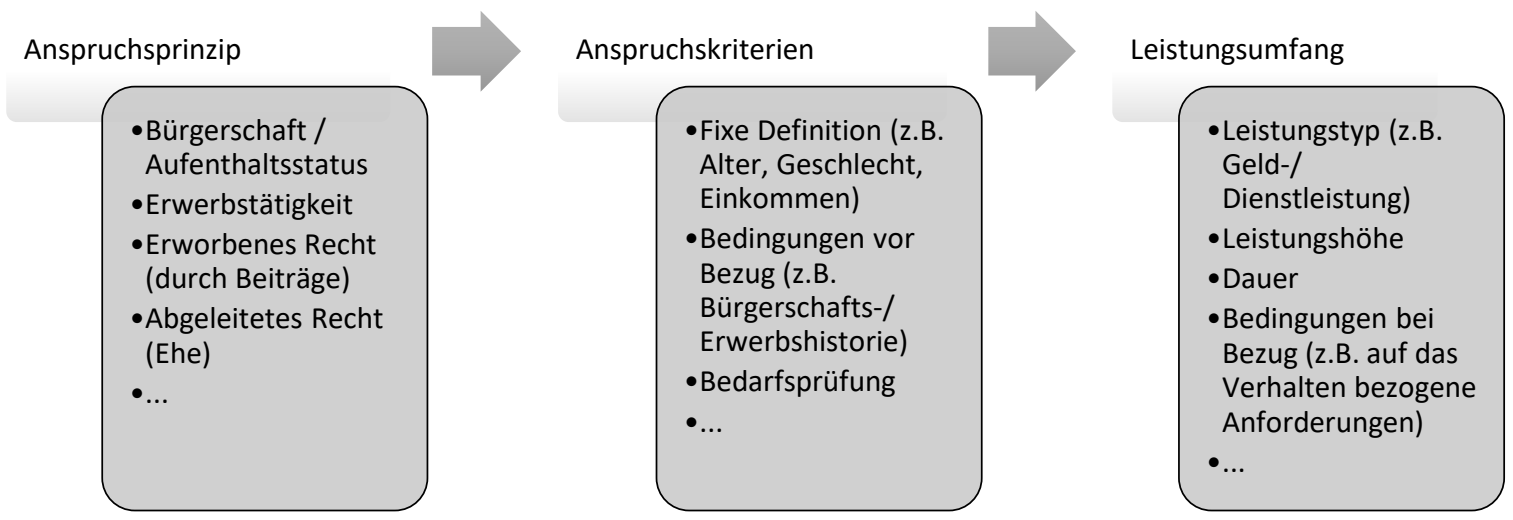

Quelle: Eigene Darstellung auf Grundlage von Blank 2011, S. 55; vgl. Clasen/Clegg 2007

Die ersten zwei Dimensionen betrachten, wem und unter welchen Umständen ein soziales Recht gewährt wird. Dies umfasst das Anspruchsprinzip und die Anspruchskriterien, d.h. die Bedingungen für die Ausübung der Rechte. Diese stellen einen zentralen Wohlfahrtsstaatsindikator dar, der die Regeln zu den bereitgestellten Leistungen ergänzt (Clasen/Clegg 2007). Letztere werden von der dritten Dimension erfasst, dem eigentlichen Inhalt der sozialen Rechte, d.h. dem Leistungsumfang. Wie Abbildung 1 zeigt, sind hierin typischerweise auch programmbezogene Auflagen enthalten (Blank 2011), die erfüllt werden müssen, um (weiterhin) eine Leistung (in voller Höhe) zu empfangen. Dies kann z.B. verhaltensbezogene Anforderungen umfassen, wenn es etwa verboten ist, öffentlich bezuschusste Kinderbetreuung in Anspruch zu nehmen, um ein Betreuungsgeld zu erhalten.

\subsection{Elterngeldrechte - Ein Typologie}

Der mehrdimensionale Charakter von Elterngeldpolitiken bringt zusätzliche Komplexität in die Analyse. Einerseits bestehen Elterngeldansprüche normalerweise aus verschiedenen Elementen, die im Prinzip alle betrachtet werden müssten, z.B. Dauer, Leistungshöhe und Flexibilität der Inanspruchnahme (Javornik 2014; Blum et al. 2017). Andererseits können sich Elterngeldrechte und ihr Umfang auch für verschiedene Gruppen innerhalb eines Landes unterscheiden (McKay et al. 2016). Insbesondere kann der Leistungsumfang auf verschiedenen Anspruchsprinzipien und kriterien basieren. Da der Leistungsumfang von Elterngeldansprüchen schon recht gut (vergleichend) erforscht ist (Ray et al. 2010; Ciccia/Verloo 2012; Javornik 2014), konzentrieren wir uns auf die Frage, wem diese Ansprüche überhaupt zur Verfügung stehen und unter welchen Bedingungen (Anspruchsprinzipien und -kriterien). Hierdurch können wir indirekt auch Schlussfolgerungen in Hinblick auf entsprechende Unterschiede beim Leistungsumfang ziehen. So haben z.B. McyKay et al. (2016) für den kanadischen Fall gezeigt, wie in einem Land, in dem Elterngeldansprüche an strikte erwerbsorientierte Bedingungen geknüpft sind, der Leistungszugang stark von der Qualität der Arbeitsmarktpartizipation der Eltern abhängt. Eltern in atypischen Beschäftigungsverhältnissen können hier nur schwer Zugang erlangen. Blofield und Martinez Franzoni (2015) heben - v.a. mit 
Blick auf lateinamerikanische Länder - außerdem hervor, dass insbesondere Frauen aus niedrigen Einkommensgruppen von Leistungen ausgeschlossen bleiben, die in der Regel eine instabilere Arbeitsmarktbindung aufweisen und auch weniger Zugang zu anderen Ressourcen haben, die sie bei der Sorgearbeit entlasten könnten. Vor diesem Hintergrund dienen uns die Anspruchsprinzipien und -kriterien als zentrale Indikatoren, um soziale Ungleichheiten von Elterngeldansprüchen zu identifizieren. Im Folgenden werden wir auf Basis der Literatur die wichtigsten Anspruchsprinzipien und -kriterien identifizieren.

\subsubsection{Anspruchsprinzipien für soziale Rechte auf Elterngeld}

Auf dem Feld der Elterngeldpolitiken existieren zwei zentrale Anspruchsprinzipien: Rechte gründen entweder auf dem Bürgerstatus ${ }^{6}$ (bzw. Aufenthaltsstatus) oder auf Erwerbstätigkeit. Die Dimension des Anspruchsprinzips gibt also Auskunft darüber, inwiefern Erwerbstätigkeit überhaupt Voraussetzung für Elterngeldansprüche ist; ob also auch nicht-erwerbstätige Personen Zugang zu Geldleistungen haben oder nicht. In Hinblick auf soziale Ungleichheiten ist diese Dimension jedoch als ambivalent zu betrachten. So folgen Elterngeldansprüche, die auf dem Bürgerstatus gründen, typischerweise einer sogenannten "Cash-for-care"-Logik und gehen mit (niedrigen) Pauschalleistungen einher (z.B. Blum et al. 2017). Sie werden unabhängig von Erwerbstätigkeit „für alle" gewährt, begünstigen jedoch eine geschlechtsspezifische Arbeitsteilung ${ }^{7}$ und richten sich in erster Linie an Mütter. Ebenfalls ist aus der bisherigen Forschung gut bekannt, dass Rechte, die "auf dem Papier" geschlechtsneutral ausgestaltet sind (indem sie z.B. als „Familienanspruch“ gewährt werden) weniger wirksam in Hinblick auf eine geschlechtergerechte Arbeitsteilung sind als individualisierte Elternzeitansprüche, d.h. Ansprüche, die explizit jedem der beiden Elternteile gewährt werden oder einen "Gender-Equality-Bonus" enthalten (Ray et al. 2010; Dearing 2016). Wie die Elternzeitregelungen auf die geschlechtliche Aufteilung von Erwerbs- und Sorgearbeit wirken, wird insbesondere durch ihre (für Männer bzw. Frauen vorgesehene) Länge sowie durch den Leistungsumfang bestimmt (für Literaturüberblicke siehe z.B. Ray et al. 2010; Dobrotić 2015). Eine paritätische Inanspruchnahme durch beide Elternteile wird insbesondere durch gut bezahlte, i.d.R. einkommensabhängige Elterngeldleistungen mit hoher Einkommensersatzrate begünstigt (z.B. Saxonberg 2013).

Elterngeldrechte, die auf Erwerbstätigkeit basieren, können ebenfalls Pauschalleistungen begründen; häufig gehen sie jedoch mit einkommensabhängigen Leistungen einher, die (während der Elternzeit) eine Einkommensersatzfunktion leisten. Zum Teil gilt für erwerbsbasierte Ansprüche der Aufenthaltsstatus als zusätzliches Anspruchskriterium. Erwerbsbasierte Systeme zielen darauf ab, die Bindung an den Arbeitsmarkt aufrechtzuerhalten. Tendenziell werden sie eher individuell geleistet, d.h. die Ansprüche gelten explizit für Männer und Frauen oder „beide Elternteile“ (siehe z.B. Blum et

\footnotetext{
${ }^{6}$ Elterngeldleistungen, die auf dem Bürgerstatus gründen, sind insofern von anderen Familienleistungen (z.B. Kindergeld) zu unterscheiden, als dass sie klar mit der entsprechenden Elternzeitleistung (als arbeitsrechtlicher Anspruch) verbunden sind. Typischerweise wird das Elterngeld an die zeitliche Dauer der Elternzeit geknüpft und es gibt Einschränkungen, inwiefern während des Bezugs (Teilzeit) gearbeitet werden darf.

${ }^{7}$ Wichtig ist hierbei auch der Hinweis der Gutachterinnen, dass über diese Leistungen auch AlleinverdienerModelle begünstigt werden - also Fälle, in denen das Einkommen eines Partners (i.d.R. des Mannes) zuzüglich der Elterngeld- und sonstigen Familienleistungen so hoch ist, dass es ausreicht eine Familie zu ernähren.
} 
al. 2017). In der Praxis finden wir häufig Mischtypen, bei denen verschiedene Elterngeldrechte für verschiedene Gruppen existieren, d.h. höhere, einkommensabhängige Leistungen für erwerbstätige Eltern und niedrigere Pauschalleistungen für nicht-erwerbstätige Eltern (z.B. in Österreich). Einkommensabhängige Elterngeldleistungen sind hier insofern speziell, als dass sie ein "verstecktes" Anspruchskriterium bereits in ihrem Leistungsumfang (oder eher: Leistungstyp) beinhalten: Selbst wenn nämlich die gesetzlich festgelegten Anspruchsprinzipien und -kriterien formal keine Erwerbstätigkeit voraussetzen, so sind doch Eltern ohne Erwerbseinkommen nicht in der Lage, dieses ökonomische Recht tatsächlich auszuüben. Daher ordnen wir einkommensabhängige Elterngeldleistungen hier per se als „erwerbsbasiert“ ein.

\subsubsection{Anspruchskriterien für soziale Rechte auf Elterngeld}

In der zweiten Dimension rücken die Anspruchskriterien ins Blickfeld, d.h. die Bedingungen, zu denen Elterngeld in Anspruch genommen werden kann. Die Anspruchskriterien können nun nacheinander für die beiden o.g. Anspruchsprinzipien betrachtet werden, d.h. Bürgerstatus sowie Erwerbstätigkeit. Die jeweiligen Kriterien können mehr oder weniger restriktiv ausgelegt sein und dementsprechend zu einem universalen oder selektiven Charakter tendieren. Universale Rechte werden hier mit Therborn (1995, S. 97) so verstanden, dass sie einen allgemeinen Anspruch aller Bürger- bzw. Einwohnerinnen zu Sozialleistungen und Einkommenssicherheit enthalten, der in erster Linie von ihrer Position im Lebensverlauf bestimmt wird. Einem Ansatz universaler sozialer Bürgerschaft folgend werden also soziale Rechte für alle bereitgestellt, um gegen bestimmte Risiken abzusichern und zwar unabhängig vom Erwerbsstatus. Es existieren jedoch auch alternative Prinzipien, darunter Residualismus, wonach soziale Rechte primär für die Bedürftigsten bereitgestellt werden, oder aber Selektivismus, wonach typischerweise spezielle Programme für unterschiedliche Klassen und Statusgruppen bestehen (Anttonen et al. 2012, S. 4-5). Gruppenspezifische soziale Rechte entsprechen einer selektiven sozialen Bürgerschaft, bei der der Leistungsumfang von Elterngeldrechten vom konkreten Erwerbsstatus der Eltern abhängt. Wenn erwerbsbasierte Policies ausschließlich auf Standarderwerbstätige ausgerichtet sind, werden soziale Ungleichheiten zwischen verschiedenen Gruppen am Arbeitsmarkt weiter verstärkt (Blofield/Martinez Franzoni 2015). Anspruchskriterien sind daher von hervorgehobener Bedeutung für die soziale Dimension von Elterngeldpolitiken.

Zunächst können wir nun diese Perspektive auf bürgerschaftsbasierte Elterngeldrechte anwenden (verstanden als Sammelbegriff, der auch (legalen) Aufenthaltsstatus einbezieht). Auch wenn bürgerschaftsbasierte Elterngeldrechte, wie im vorigen Abschnitt ausgeführt, per se als ambivalent in Hinblick auf soziale Ungleichheiten gelten müssen, so kann doch nun die Dimension der Anspruchskriterien soziale Ungleichheiten innerhalb dieser Modelle beleuchten (z.B. „Ausschluss“" von Hartz-IV-Empfängern gegenüber anderen ökonomisch Inaktiven im deutschen Fall). Denn es wird ersichtlich, dass bürgerschaftsbasierte Rechte typischerweise an weitere Kriterien gebunden sind, z.B. die zeitliche Dauer und/oder den Status des Aufenthalts in einem Land. Indem ein vorhergehender Wohnsitz vorausgesetzt wird, kann der Zugang zu Elterngeldrechten eingeschränkt

\footnotetext{
${ }^{8}$ Nicht formal, wohl aber de facto sind Hartz-IV-Empfängerinnen in Deutschland vom Elterngeldbezug ausgeschlossen, da es seit 2010 auf ihre Grundsicherungsleistungen voll angerechnet wird (in den Jahren zuvor war dies nicht der Fall) (vgl. Reimer et al. 2017).
} 
und damit ein selektives System produziert werden. Innerhalb der EU betrifft dies vor allem Migranten aus Nicht-EU-Ländern (s.o.). Zudem können Einkommensprüfungen vorgenommen werden, um über Zugang oder Ausschluss von sozialen Rechten zu entscheiden. Bedarfsprüfungen erzeugen häufig residuale Systeme, in denen soziale Rechte den bedürftigsten Einwohnerinnen vorbehalten sind (Anttonen et al. 2012).

Betrachten wir nun in einem zweiten Schritt die Anspruchskriterien für erwerbsbasierte Elterngeldrechte, so ist festzuhalten, dass diese an die Länge der vorherigen Erwerbshistorie oder Sozialversicherungszeiträume gebunden sein können. Ebenfalls können, wie auch für bürgerschaftsbasierte Leistungen, (zusätzliche) Bedarfsprüfungen stattfinden. Je länger der Zeitraum ist, der vorausgesetzt wird, um für soziale Rechte zu qualifizieren (v.a. wenn er ohne Unterbrechungen oder beim gleichen Arbeitgeber erfolgt sein muss), desto eingeschränkter ist der Zugang zu erwerbsbasierten Leistungen, v.a. für Eltern mit weniger stabilen Erwerbsverläufen (z.B. Saisonarbeit, befristete Verträge). Anspruchskriterien können auch den Leistungsumfang regulieren, wenn z.B. die Leistungshöhe in Abhängigkeit von der vorherigen Erwerbsdauer ansteigt. Es existieren noch eine Reihe weiterer Anspruchskriterien für erwerbsbasierte Leistungen. So können sich erwerbsbasierte Anspruchskriterien zwischen dem öffentlichen Dienst und der Privatwirtschaft unterscheiden oder auch Unterschiede zwischen Arbeitsmarktsektoren bestehen, z.B. aufgrund von Kollektivverträgen oder freiwilligen betrieblichen Sozialleistungen. Auch können Selbstständige oder geringfügig Beschäftigte (teilweise) von den Leistungssystemen ausgeschlossen sein. Hierdurch werden die Ungleichheiten, die zwischen verschiedenen Erwerbsformen im Zugang zu Elterngeld (und dessen Leistungsumfang) bestehen, weiter verstärkt. Und schließlich unterscheiden sich die Länder auch dahingehend, ob Studierende oder Langzeitarbeitslose Zugang zu (gleich hohen) Elterngeldzahlungen besitzen (vgl. z.B. Blum et al. 2017). Auch hier kann von einem stärker universalen oder selektiven Charakter von Elternzeitrechten gesprochen werden, der durch die vorhandenen Anspruchskriterien geprägt wird. Angesichts der zunehmenden Zahl prekärer Beschäftigungsverhältnisse und unterbrochener Erwerbsverläufe (Vaughan-Whitehead 2012) können wir vor diesem Hintergrund annehmen, dass in Ländern, in denen solche strikten Anspruchskriterien an die Form der Erwerbstätigkeit gerichtet werden, eine zunehmende Zahl von Personen vom Zugang zu Elterngeld ausgeschlossen bleibt.

\subsubsection{Eine Typologie sozialer Rechte auf Elterngeld}

Betrachtet man die o.g. Unterscheidungen, so bildet sich eine Klassifikation von vier verschiedenen Anspruchstypen auf Elterngeldrechte heraus (Abb. 2). Wenn die Rechtsansprüche dem Prinzip des Bürgerstatus folgen und die Anspruchskriterien nicht strikt sind (z.B. ein legaler Aufenthalt erst ab dem Tag der Geburt vorausgesetzt wird), so können wir von einem universalen Elternschafts-Modell sprechen. Universal bezieht sich hier auf den Deckungsgrad des sozialen Rechts. Entsprechend kann man, wenn die Anspruchskriterien nicht strikt sind, aber die Leistung auf Erwerbstätigkeit gründet (z.B. jede Form von Erwerbsverhältnis, die am Tag der Geburt besteht), von einem universalen AdultWorker-Model sprechen. Der Begriff des Adult Worker Model geht auf Lewis (2001) zurück; sie beschreibt damit Policies, die von der Prämisse ausgehen, dass alle Erwachsenen am Arbeitsmarkt aktiv sind, während die Sorgearbeit kommodifiziert und de-familialisiert wird. 
Wie weiter oben beschrieben wurde, können Anspruchskriterien auch einen selektiven - oder sogar residualen - Charakter aufweisen. In diesen Fällen kann von einem selektiven Modell gesprochen werden, d.h. Leistungen sind auf bestimmte sozioökonomische Gruppen ausgerichtet und soziale Programme unterscheiden sich zwischen Statusgruppen. Dementsprechend kann ein selektives Elternschafts-Modell identifiziert werden, wenn strikte Anspruchskriterien mit einem am Bürgerstatus orientierten Anspruchsprinzip einhergehen (z.B. wenn der Aufenthaltsstatus für mindestens ein Jahr vor der Geburt erfüllt sein muss). Und schließlich kann von einem selektiven Adult-Worker-Modell gesprochen werden, wenn eine Kombination von strengen Anspruchskriterien und erwerbsbasiertem Anspruchsprinzip aufzufinden ist (z.B. Voraussetzung einer ununterbrochenen Beschäftigung von mindestens einem Jahr vor der Geburt). Länder, die in Richtung eines selektiven Modells tendieren, sind durch größere soziale Ungleichheiten bei den Elterngeldrechten gekennzeichnet, indem z.B. der Zugang zu (generöseren) Rechten Eltern mit starker Arbeitsmarktbindung oder längerem Bürgerstatus vorbehalten ist. Wenn Bedarfsprüfungen zusätzlich zu bestehenden Anspruchskriterien durchgeführt werden, nimmt ein selektives Modell residualen Charakter an, d.h. Elterngeldansprüche werden allein an die bedürftigsten Einwohner bzw. Arbeitnehmer adressiert.

Abbildung 2: Eine Typologie von Elterngeldrechten

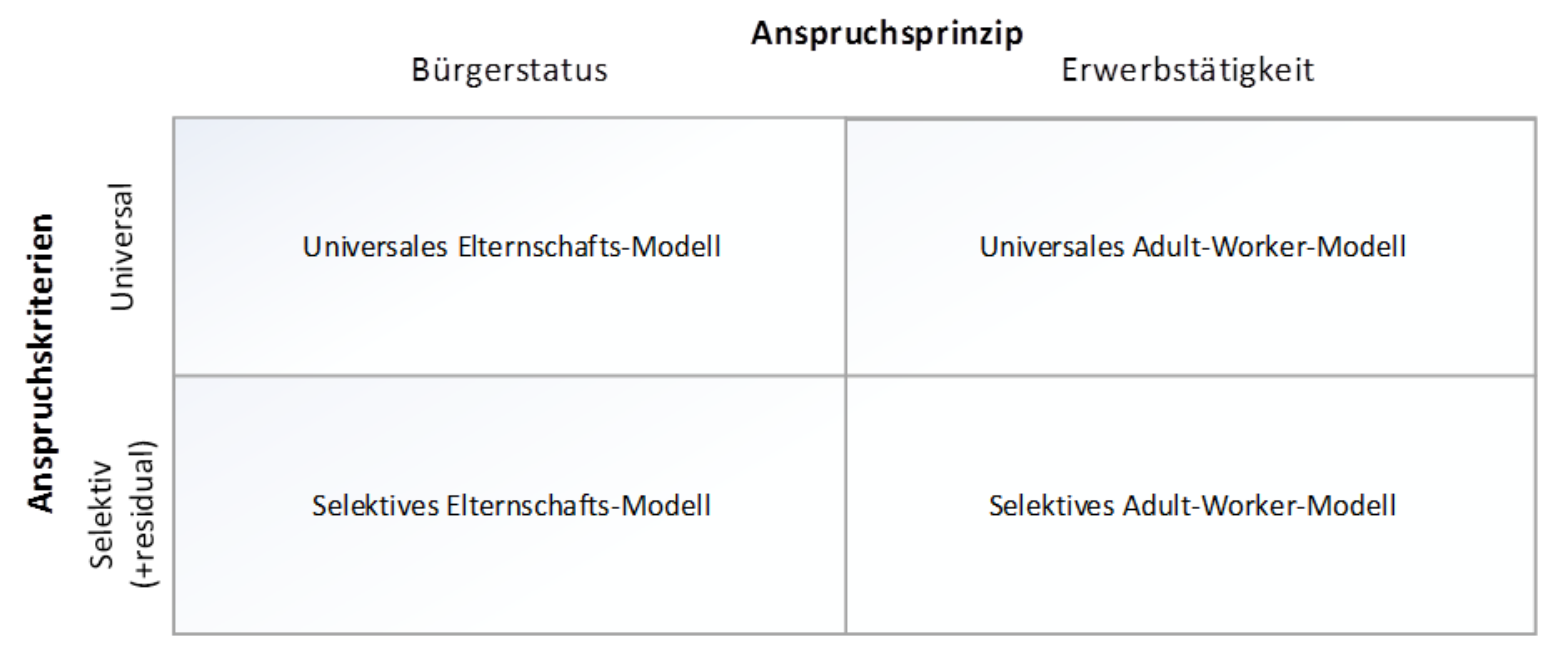

Quelle: Eigene Darstellung

Wie wir im folgenden Abschnitt näher ausführen werden, finden sich in der Realität nur selten Entsprechungen dieser vier Idealtypen. Das ist u.a. darauf zurückzuführen, dass sich die Länder zunehmend in Richtung von Mischsystemen bewegt haben; bürgerschaftsbasierte Elterngeldleistungen (als Basisleistung) werden dann mit (generöseren) erwerbsbasierten Elterngeldleistungen verknüpft (Dobrotić/Blum 2019). Während nach wie vor eine Reihe von rein erwerbsbasierten Modellen existieren, ist dies für bürgerschaftsbasierte Modelle nicht (mehr!) der Fall. Die aufzufindenden Anspruchskriterien variieren von universal bis residual, wobei sich auch innerstaatliche Varianzen zwischen dem öffentlichen Dienst und der Privatwirtschaft, zwischen Angestellten und Selbstständigen oder zwischen verschiedenen Arbeitsmarktsektoren zeigen. 


\section{Illustration anhand von Länder-Kurzstudien}

Um die Typen von Anspruchsberechtigungen empirisch zu illustrieren, greifen wir auf Informationen aus dem Annual Review of Leave Policies and Related Research (Blum et al. 2017) zurück. Diese Informationen haben wir mit der OECD Family Database (OECD 2016), der MISSOC Datenbank (2006, 2016) sowie nationalen Quellen abgeglichen. Aus Perspektive sozialer Rechte konzentrieren wir uns hier auf gesetzliche Ansprüche auf nationalstaatlicher Ebene. Davon unabhängig sind in einigen Ländern auch regionale oder lokale Leistungen von gewisser Bedeutung (z.B. Deutschland, Spanien). Auch kann es Zusatzleistungen aus Kollektivverträgen (z.B. Dänemark, Italien, Niederlande) oder als individuelle Arbeitgeberleistung geben (z.B. Großbritannien, Kroatien). Auch wenn diese Zusatzleistungen in einigen Ländern für den Zugang zu Elterngeldleistungen bedeutsam sein mögen, handelt es sich nicht um soziale Rechte solange kein gesetzlicher Anspruch auf diese Leistungen besteht (vgl. Blank 2011).

Im Folgenden werden drei Fälle vorgestellt, um die Logik der oben identifizierten Idealtypen (bzw. ihrer möglichen Kombinationen) zu veranschaulichen. Sie zeigen die breite Varianz an Anspruchsprinzipien und -kriterien im internationalen Vergleich. Die deutsche Regelung wird hierbei im Vergleich mit Belgien und Kroatien betrachtet. Zusammenfassend haben zwei der drei Länder (nämlich: Deutschland und Kroatien) einen Wandel in Richtung eines Mischsystems vollzogen, wobei Deutschland von einem bürgerschaftsbasierten Modell ausging, Kroatien hingegen von einem erwerbsbasierten Modell. Innerhalb der heutigen Mischsysteme tendiert Deutschland durch seine Anspruchskriterien zu einem universalen Modell, Kroatien zu einem selektiven Modell. Belgien hingegen kommt dem Idealtypus eines selektiven Adult-Worker-Modells sehr nahe.

\subsection{Deutschland: Vom selektiven Elternschafts-Modell zum universalen Mischsystem}

Elternzeit (bis 2001: Erziehungsurlaub) und die entsprechende Geldleistung (Erziehungsgeld) wurden in Deutschland 1986 als bürgerschaftsbasierte Rechte eingeführt. Nach mehreren Reformen, die zu einer verlängerten Anspruchsberechtigung führten, existierte 2006 eine Pauschalleistung von monatlich €300, die für die Dauer von zwei Jahren gezahlt wurde und unabhängig von einer vorherigen Erwerbstätigkeit war. Es handelte sich um einen Familienanspruch, der also im Zugang (nicht aber in Hinblick auf seine Wirkung) geschlechtsneutral ausgestaltet war. Es wurde außerdem eine Einkommensprüfung durchgeführt, durch die das Erziehungsgeld entsprechend dem Haushaltseinkommen reduziert wurde oder ganz entfallen konnte. Es kann also für den Zeitraum bis 2006 ein selektives Elternschafts-Modell identifiziert werden. 2007 wurde das System grundlegend reformiert. Es wurde ein einkommensabhängiges Elterngeld eingeführt, das einen Einkommensersatz von etwa 65 Prozent des vorherigen Einkommens bietet (Reimer et al. 2017). Jedoch wurde die bürgerschaftsbasierte Variante nicht abgeschafft: Es existiert nach wie vor eine Basisleistung in Höhe von $€ 300$, die unabhängig von einer vorherigen Erwerbstätigkeit gezahlt wird (z.B. an Hausfrauen), nun allerdings nur noch für die Dauer eines Jahres. Auch wenn ein bestehendes Erwerbsverhältnis keine offizielle Anspruchsvoraussetzung darstellt, um Zugang zum Elterngeld zu erhalten, können wir dennoch von einem Mischsystem aus erwerbs- und bürgerschaftsbasierten Leistungen sprechen. Denn das einkommensabhängige Elterngeld wäre de facto für nicht-erwerbstätige Eltern nicht zugänglich; diesen Zugang erhalten sie erst über das speziell eingerichtete Mindestelterngeld von $€ 300$ (BEEG §2). 
Beim Elterngeld handelt es sich um einen Familienanspruch von 14 Monaten, von denen zwei Monate exklusiv für jeden Elternteil reserviert sind und bei Nicht-Inanspruchnahme verfallen (sogenannte Väter- oder Partnermonate). Das System weist demnach einen gender-sensiblen Zugang auf, d.h., es enthält einzelne Elemente, die einer geschlechtsspezifischen Inanspruchnahme entgegensteuern sollen. Dieser gender-sensible Zugang wurde mit dem Elterngeld Plus (2015) weiter gestärkt. ${ }^{9}$ Regionale Varianzen bestehen insofern, als dass zwei Bundesländer (Bayern, Sachsen) ein einkommensgeprüftes Landeserziehungsgeld für zweijährige Kinder zahlen (d.h., sie verlängern die Geldzahlung für das letzte Jahr der Elternzeit, die ja mit drei Jahren die maximale Elterngeldbezugsdauer übersteigt).

Betrachtet man die Anspruchskriterien, so ist das deutsche System mit der 2007er Reform universaler geworden, da nun keine Einkommensprüfungen mehr stattfinden. Ein Arbeitsverhältnis zum Zeitpunkt der Geburt begründet eine Anspruchsberechtigung für (einkommensabhängige) Leistungen; eine vorherige Erwerbsperiode ist jedoch keine Voraussetzung. Auch Selbstständige sind im System eingeschlossen. Ein Wohnsitz in Deutschland ist Voraussetzung für den Leistungsbezug, allerdings mit Ausnahmen (z.B. für EU-Wanderarbeitnehmer, Adoptiveltern). Nicht-EU-Einwohner sind anspruchsberechtigt, wenn sie eine permanente Aufenthaltserlaubnis oder einen Wohnsitz in Deutschland besitzen. Asylbewerber haben frühestens nach dreijährigem Aufenthalt Anspruch auf Elterngeld. Seit 2011 wird das Mindestelterngeld (€300) auf die Sozialhilfeleistungen angerechnet: Langzeitarbeitslose erhalten zwar das Mindestelterngeld, gleichzeitig wird aber ihre Sozialhilfe um eben diese Summe gekürzt. De facto wurde innen das Elterngeld also seit 2011 gestrichen, während es zuvor ausgezahlt worden war (Reimer et al. 2017). Dies reduziert den inklusiven Charakter eines ansonsten universalen Mischsystems aus erwerbs- und bürgerschaftsbasierten Elterngeldleistungen.

\subsection{Belgien: Ein selektives Adult-Worker-Modell}

In Belgien bestehen ausschließlich erwerbsbasierte Elterngeldleistungen. Der Mutterschutz beträgt 15 Wochen für erwerbstätige Mütter (zwölf Wochen für selbstständige Mütter) und es existiert auch eine Väterzeit von zehn Arbeitstagen. Die Elternzeit von vier Monaten ist ein individueller Anspruch, der nicht zwischen Mutter und Vater übertragbar ist. Belgien weist also ein entgendertes Modell im Bereich des Elterngelds auf. Allerdings existiert kein einkommensabhängiges Elterngeld für den Zeitraum der Elternzeit, sondern eine Pauschalleistung in Höhe von monatlich $€ 702$ netto. Dieser Betrag wiederum wird nur für Vollzeit erwerbstätige Eltern gezahlt, während in Teilzeit erwerbstätige Eltern eine niedrigere Leistungshöhe entsprechend ihrem Arbeitsumfang erhalten. Umgekehrt wird auch die Leistungshöhe reduziert, wenn Eltern einen Elterngeldbezug mit Teilzeiterwerbstätigkeit kombinieren. Das belgische Modell weist regionale Varianzen auf: In Flandern wird eine Zusatzleistung gezahlt, deren Höhe sich zwischen den Erwerbssektoren unterscheidet und im NonProfit-Sektor am höchsten liegt (Merla 2017). Die Segmentierung entlang von Erwerbssektoren, die sich in dieser regionalen Elterngeldvariante zeigt, ist gemeinsam mit der Segmentierung entlang von

\footnotetext{
${ }^{9}$ Eltern können nun wählen, ob Sie das Elterngeld Plus anstelle des klassischen Elterngelds beziehen oder es sukzessive mit dem Elterngeld kombinieren. Es ersetzt einen Teil des Einkommensausfalls, wenn Eltern von unter zweijährigen Kindern eine Teilzeiterwerbstätigkeit mit dem Elterngeldbezug kombinieren (um Elternzeit und -geld in Anspruch zu nehmen dürfen Eltern allerdings nicht mehr als 30 Stunden pro Wochen erwerbstätig sein). Weiterhin wird ein Gender-Equality-Bonus gezahlt, wenn beide Elternteile für mindestens vier aufeinander folgende Monate zwischen 25 und 30 Wochenstunden arbeiten (Reimer et al. 2017).
} 
Erwerbsformen typisch für die Anspruchsberechtigungen im belgischen Fall. Anspruch auf Elterngeld haben hier nur Personen, die innerhalb der letzten 15 Monate vor Beginn der Elternzeit mindestens zwölf Monate für denselben Arbeitgeber gearbeitet haben. Arbeitnehmern, die diese Anspruchskriterien nicht erfüllen, können Arbeitgeber die Elternzeit per Vereinbarung gewähren (Merla 2017). Weiterhin bestehen Unterschiede zwischen dem öffentlichen Dienst und der Privatwirtschaft; im öffentlichen Dienst Beschäftigte müssen das Anspruchskriterium der einjährigen Erwerbstätigkeit für den aktuellen Arbeitgeber nämlich nicht erfüllen. Auch sind Selbstständige weder für die Elternzeit noch den Elterngeldbezug anspruchsberechtigt. Ähnlich selektive Anspruchsberechtigungen für erwerbsbasierte Leistungen finden sich z.B. auch bei dem belgischen Zeitkredit (Leitner 2005), einer (niedrig entlohnten) Laufbahnunterbrechung, die prinzipiell im Anschluss an die Elternzeit genutzt werden könnte - was aber realiter kaum erfolgt, der Zeitkredit wird vornehmlich als "Altersteilzeit“ beim Übergang in den Ruhestand verwendet (Merla 2017). Vor diesem Hintergrund kommt Belgien dem Idealtyp eines selektiven Adult-Worker-Modells recht nahe.

\subsection{Kroatien: Vom selektiven Adult-Worker-Modell zum (selektiven) Mischsystem}

Bezahlte Elternzeit (sog. „Zusätzlicher Mutterschutz“) wurde in Kroatien erstmals 1978 im Rahmen eines erwerbsbasierten Systems eingerichtet. Bürgerschaftsbasierte Pauschalleistungen wurden Mitte der 1990er Jahre eingeführt - zunächst ausschließlich für Eltern von Zwillingen oder Eltern von drei und mehr Kindern, später, Anfang der 2000er Jahre, für alle Eltern unabhängig von der Kinderzahl. Damit hat Kroatien einen inkrementellen Wandel in Richtung eines Mischsystems durchschritten, in dem inklusivere bürgerschaftsbasierte sowie erwerbsbasierte Elterngeldrechte koexistieren. Ebenso hat es sich von einem klar gegenderten System weiterentwickelt und weist nun einige entgenderte Elemente auf: So wurde 2009 das erwerbsbasierte Elterngeld, das ursprünglich ausschließlich als Recht der Mutter definiert war, in ein individuelles, aber übertragbares Recht beider Elternteile transformiert. 2013 wurde ein nicht-übertragbares Elterngeld eingeführt, das allerdings nur für Familien mit zwei erwerbstätigen Elternteilen und ein oder zwei Kindern verfügbar ist. Nach dem sieben Monate dauernden Mutterschutz hat jeder (selbstständig oder unselbstständig) erwerbstätige Elternteil einen Rechtsanspruch auf vier Monate einkommensabhängiges Elterngeld, wobei zwei dieser Monate nicht an den anderen Elternteil übertragbar sind. Allerdings bleibt das Elterngeld nach wie vor übertragbar für Eltern von Zwillingen sowie Eltern von drei oder mehr Kindern, die außerdem einen Anspruch auf längere Elternzeit besitzen (15 Monate). Erwerbstätige Väter sind nur dann anspruchsberechtigt, wenn auch die Mutter erwerbstätig ist; da außerdem ein recht niedriger Höchstbetrag des Elterngelds existiert $(€ 353,60)$ nehmen in erster Linie Mütter Elternzeit. Im erwerbsbasierten System wird ein pauschales Elterngeld gezahlt bis das Kind ein Jahr alt ist (oder drei Jahre im Fall mehrerer Kinder). Diese Pauschalleistung geht vorrangig an Mütter und kann nur dann an den Vater übertragen werden, wenn die Mutter eine Erwerbstätigkeit aufnimmt oder (z.B. gesundheitlich) nicht in der Lage ist, sich um das Kind zu kümmern (Dobrotić 2015). Da also die Geldleistung nur unter bestimmten Bedingungen an den Vater übertragen werden kann, weist das kroatische System einen gegenderten Zugang auf - insbesondere für Frauen mit niedriger Arbeitsmarktbindung.

Seit 2016 existieren auch regionale Varianzen: Die Stadt Zagreb hat einen sogenannten „ElternBetreuer"-Status eingeführt, d.h. einen Rechtsanspruch auf eine vergleichsweise sehr generöse 
Geldleistung (nämlich $€ 520$ pro Monat, was $150 \%$ des Mindestlohns entspricht). Diese Geldleistung steht für arbeitslose Eltern mit drei oder mehr Kindern zur Verfügung (darunter mindestens einem Kind im Vorschulalter), wenn sie die kroatische Staatsbürgerschaft haben und seit mindestens fünf Jahren vor der Geburt ununterbrochen in der Stadt Zagreb gelebt haben. Während des Bezugs dieser Geldleistung darf kein Platz in einer Kinderbetreuungseinrichtung in Anspruch genommen werden; die Leistung kann bezogen werden, bis das jüngste Kind in der Familie 15 Jahre alt wird (Dobrotić 2017).

Mit Blick auf die Anspruchskriterien haben alle erwerbstätigen Eltern, die krankenversichert sind, Anspruch auf das einkommensabhängige Elterngeld. Wenn sie allerdings nicht innerhalb der letzten zwei Jahre mindestens 12 Monate ununterbrochen oder mindestens 18 Monate mit Unterbrechungen erwerbstätig gewesen sind, sind sie lediglich für die niedrigere, bürgerschaftsbasierte Pauschalleistung qualifiziert. Auch Eltern ohne regulären Arbeitsvertrag (z.B. Freischaffende) haben nur Anspruch auf die bürgerschaftsbasierte Leistung. Diese Pauschalleistungen stehen für alle kroatischen Bürger zur Verfügung. Ausländer mit permanentem Aufenthalt in Kroatien sind anspruchsberechtigt, wenn sie drei Jahre (Eltern ohne regulären Arbeitsvertrag, registrierte Arbeitslose, Bauern) bzw. fünf Jahre (nicht-erwerbstätige Eltern, Studierende) vor der Geburt ununterbrochen im Land gelebt haben. Dieser Aufenthaltszeitraum gilt jedoch nicht für Asylberechtigte und subsidiär Schutzberechtigte. Arbeitslose Personen müssen überdies die Bedingung erfüllen, innerhalb der zwei Jahre vor der Geburt für mindestens neun Monate oder mindestens zwölf Monate mit Unterbrechungen offiziell arbeitslos gemeldet oder aber vorab für mindestens sechs Monate beschäftigt gewesen zu sein. Es lässt sich schlussfolgern, dass Kroatien ein komplexes Mischsystem aus selektiven erwerbs- und bürgerschaftsbasierten Elterngeldleistungen aufweist, das in Hinblick auf die Gender-Dimension als nach wie vor als ambivalent gelten kann.

\section{Elterngeldrechte: Nutzen und Grenzen der Typologie}

Abschließend möchten wir diskutieren, wie die vorgeschlagene Typologie von Anspruchsberechtigungen auf Elterngeld angewendet werden kann, was sie - im Verhältnis zu bestehenden Typologien - beitragen kann und wo ihre Grenzen liegen.

Wie oben diskutiert, werden bürgerschaftsbasierte Leistungen zwar unabhängig von Erwerbstätigkeit „für alle" Bürgerinnen bereitgestellt, gleichzeitig sind sie in Hinblick auf soziale Ungleichheiten als ambivalent zu betrachten. Denn insbesondere wenn sie einen Anspruch auf ein lang gezahltes, niedriges Pauschal-Elterngeld begründen, das formal „geschlechtsneutral“ ausgestaltet ist, befördern sie eine geschlechtsspezifische Arbeitsteilung, die sich - wiederum aus Perspektive sozialer Ungleichheiten - auch nur bestimmte Familien „leisten“ können (Leitner 2003; Javornik 2014).

Die Hinzunahme der zweiten Dimension sozialer Rechte, nämlich der Anspruchskriterien, erlaubt vor diesem Hintergrund einen tiefgehenden Einblick in die sozialen Ungleichheiten beim Leistungszugang für beide Modelle, den bürgerschafts- und den erwerbsbasierten Ansatz. Die hier vorgeschlagene Typologie kann somit einen wichtigen Beitrag zur vergleichenden Literatur zu Elterngeldregelungen leisten, v.a. da diese bislang recht stark auf die Dimension des Leistungsumfangs fokussiert geblieben ist. Aus Perspektive sozialer Rechte kann identifiziert werden, welche Gruppen weniger Rechte 
haben oder ganz vom Leistungsbezug ausgeschlossen sind. Es wurde gezeigt, wie selektive erwerbsbasierte Systeme zu einer Ungleichbehandlung von Personen mit mehr oder weniger „sicherem“ Arbeitsverhältnis führen, wobei v.a. Personen in atypischen Beschäftigungsverhältnissen oder mit weniger geradlinigen Erwerbsverläufen benachteiligt sind. Aber auch bei erwerbsbasierten Elterngeldleistungen kann der Zugang eingeschränkt werden. So können bestimmte Gruppen benachteiligt sein (z.B. Langzeitarbeitslose, Selbstständige) oder der Zugang zu bürgerschaftsbasierten Rechten kann an eine lange Aufenthaltsdauer im Land geknüpft werden (z.B. drei bis fünf Jahre in Kroatien). Allerdings bleibt die vorgeschlagene Typologie bislang darauf fokussiert, wer auf welcher Basis für den Elterngeldbezug qualifiziert ist, also auf Anspruchsprinzipien und -kriterien. Unsere Typologie kann daher die „versteckten“ Ungleichheiten im Zugang zu sozialen Rechten beleuchten, und damit Unterschiede zwischen verschiedenen sozioökonomischen Gruppen und für verschiedene Anspruchsbedingungen (z.B. Erwerbsform, öffentlicher Dienst und Privatwirtschaft, Erwerbssektoren) deutlich machen. Aber es fehlt (bislang) ein systematischer Bezug zur dritten Dimension sozialer Rechte, dem Leistungsumfang. Auf Basis der Typologie sind allerdings erste Rückschlüsse möglich, inwiefern verschiedene Anspruchsprinzipien und -kriterien einen bestimmten Leistungsumfang nach sich ziehen. Beispielsweise sind für Personen mit instabilen Erwerbsverläufen typischerweise niedrigere (Pauschal-)Leistungen vorgesehen oder diese werden vom Leistungsbezug ausgeschlossen (z.B. Belgien). Da unser Schwerpunkt hier auf dem Arbeitsmarktbezug lag, haben wir auch nicht systematisch andere Anspruchsbedingungen in den Blick genommen, z.B. Familienstatus oder Kinderzahl. Und schließlich bestehen viele andere sozioökonomische, kulturelle und institutionelle Faktoren, die das „Anspruchsgefühl“ der Eltern und ihre Fähigkeit, die Rechte auch tatsächlich auszuüben, beeinflussen, z.B. Geschlechternormen am Arbeitsplatz und in der Gesellschaft (Hobson et al. 2011; Kurowska 2016).

Abschließend erscheint es wichtig, danach zu fragen, welchen Status die hier vorgeschlagene Typologie gegenüber anderen Typologien einnehmen kann, die auf dem Feld der Elterngeldforschung Verwendung finden. Da wir diese Frage hier nicht systematisch in den Blick nehmen können, wollen wir lediglich einige zentrale Punkte nennen, die aus unserer Sicht Anknüpfungsstellen für weitere Forschung bieten können. Wir teilen das Argument von Saxonberg (2013, S. 26), dass sich Typologien auf Policies konzentrieren sollten, nicht auf deren Auswirkungen. Diese Unterscheidung gewinnt an Bedeutung, wenn durch Forschung etwas erklärt werden soll - entweder die Wirkungen bestimmter Policies oder aber die Determinanten der Policies selbst. Die wohl am häufigsten für die vergleichende Familienpolitikforschung verwendete Typologie (Kurowska 2016) ist die Dichotomie zwischen Familialisierung und De-Familialisierung (Esping-Andersen 1999). Sie wurde v.a. von Leitner (2003) durch die Unterscheidung von expliziter und impliziter (De-)Familialisierung weiter verfeinert. Diese Typologie hat v.a. aus Gender-Perspektive Anwendung gefunden, wenn danach gefragt wird, inwiefern (Elterngeld-)Politiken die geschlechtliche Arbeitsteilung zwischen Sorge- und Erwerbsarbeit beeinflussen.

Die in diesem Beitrag vorgeschlagene Perspektive sozialer Rechte ermöglicht einen umfassenderen Blick auf die verschiedenen Dimensionen von Elterngeldpolitiken. Gender-orientierte Klassifikationen und Vergleiche tendieren dazu, die erste und dritte Dimension sozialer Richte in den Blick zu nehmen. In kritischer Auseinandersetzung mit Esping-Andersens Konzept der (De-)Kommodifizierung wird häufig das Anspruchsprinzip betrachtet, aber auch ein starker Schwerpunkt auf den Leistungsumfang gelegt, z.B. Dauer der Elternzeit und monatliche Elterngeldhöhe (z.B. Leitner 2003; Ciccia/Verloo 2012; Saxonberg 2013; Dearing 2016). Die Frage, wer denn eigentlich Anspruch auf 
diese Leistungen hat, wird dann typischerweise nur auf die jeweiligen Rechte von Frauen und Männern ausgeweitet (Familienanspruch vs. individueller Rechtsanspruch, Übertragbarkeit, etc.). Die Anspruchskriterien hingegen werden nicht systematisch einbezogen, auch wenn sie zentral sind um den Zugang zu Elterngeldrechten, ihren Arbeitsmarktbezug und die mit den verschiedenen Anspruchsbedingungen einhergehenden sozialen (Un-)gleichheiten zu verstehen. Dies ist die wesentliche Stärke unserer Typologie. Für zukünftige Forschung könnte es interessant sein, sie mit der Klassifikation der (De-)Familialisierung zu kombinieren. Denn nur eine systematische Verknüpfung aller drei Dimensionen von Elterngeldrechten erlaubt es, die ungleichen Rechte zwischen verschiedenen Gruppen vollumfänglich zu verstehen (z.B. kürzerer Leistungsbezug für Selbstständige, höhere Leistungen im öffentlichen Dienst etc.). Auch wenn Elterngeldrechte hier wichtig sind, ist doch die Perspektive von universalen versus selektiven, von Adult-Worker- versus Elternschafts-Modellen darüber hinaus auch auf andere (sozialpolitische) Leistungen übertragbar.

\section{Literaturverzeichnis}

Dobrotić, I./Blum, S. (2019, i.E.): A social right? Access to leave and its relation to parents' labour market position, in: Duvander, A.-Z./Koslowski, A./Moss, P. (Hrsg.), Parental leave and beyond: recent developments, current issues, future directions, Bristol.

Anttonen, A./Haikio, L./Stefansson, K./Sipila, J. (2012): Universalism and the challenge of diversity, in: Anttonen, A./Haikio, L./Stefansson, K. (Hrsg.), Welfare State, Universalism and Diversity, Cheltenham.

Blank, F. (2007): Analyzing social rights in different contexts - a qualitative multi-dimensional approach, Paper präsentiert auf der ESPAnet Konferenz, 20.-22. September, Wien.

- (2011): Soziale Rechte 1998-2005. Die Wohlfahrtsstaatsreformen der rot-grünen Bundesregierung, Wiesbaden.

Blofield, M./Martinez Franzoni, J. (2015): Maternalism, Co-responsibility, and Social Equity: A Typology of Work-Family Policies, Social Politics, 22(1), pp. 38-59.

Blum, S./Koslowski, A./Moss, P. (2017): International Review on Leave Policies and Related Research 2017. Abrufbar unter: http://www.leavenetwork.org/lp_and_r_reports/ (4.6.2018)

Bonoli, G. (2005): The politics of new social policies: providing coverage against new social risks in mature welfare states, Policy \& Politics, 33(3), S. 431-449.

Cantillon, B. (2011): The paradox of the social investment state: growth, employment and poverty in the Lisbon era, Journal of European Social Policy, 21(5), S. 432-449.

Ciccia, R./Verloo, M. (2012): Parental Leave regulations and the persistence of the male breadwinner model: Using fuzzy-set ideal type analysis to assess gender equality in an enlarged Europe, Journal of European Social Policy, 22(5), S. 507-528.

Clasen, J./Clegg, D. (2007): Levels and levers of conditionality: Measuring change within welfare states, in: Clasen, J./Siegel, N. A. (Hrsg.), Investigating welfare state change: The 'dependent variable problem' in comparative analysis, Cheltenham. 
Dearing, H. (2016): Gender equality in the division of work: How to assess European leave policies regarding their compliance with an ideal leave model, Journal of European Social Policy, 26(3), S. 234-47.

Dobrotić, I. (2015): Politike usklađivanja obiteljskih obaveza i plaćenog rada i položaj roditelja na tržištu rada, Revija za socijalnu politiku, 22(3), S. 353-374.

- (2017): Croatia country note, in: Blum, S./Koslowski, A./Moss, P. (Hrsg.), International Review of Leave Policies and Related Research 2017. Abrufbar unter: http://www.leavenetwork.org/lp_and_r_reports/ (4.6.2018)

Esping-Andersen, G. (1999): Social Foundations of Postindustrial Economies, Oxford.

- (1990): The Three Worlds of Welfare Capitalism, Princeton.

Europäisches Parlament (2015): Arbeit und Sozialhilfe für Asylbewerber und Flüchtlinge. Ausgewählte Mitgliedsstaaten der EU. Abrufbar unter:

http://www.europarl.europa.eu/RegData/etudes/IDAN/2015/572784/EPRS_IDA(2015)572784_DE.pdf

(4.6.2018)

Ghysels, J./Van Lancker, W. (2011): The unequal benefits of activation: an analysis of the social distribution of family policy among families with young children, Journal of European Social Policy, 21(5), S. 472-485.

Hobson, B./Fahlén, S./Takács, J. (2011): Agency and capabilities to achieve a work-life balance: a comparison of Sweden and Hungary, Social Politics, 18(2), S. 168-98.

Javornik, J. (2014): Measuring state de-familialism: Contesting post-socialism exceptionalism, Journal of European Social Policy, 24(3), S. 240-257.

Kurowska, A. (2016): (De)familization and (De)genderization - Competing or Complementary Perspectives in Comparative Policy Analysis?, Social Policy \& Administration, S. 1-21.

Kvist, J. (2005): Exploring Diversity: measuring welfare state change with fuzzy-set methodology, in: Clasen, J./Siegel, N. A. (Hrsg.), Investigating welfare state change: The 'dependent variable problem' in comparative analysis, Cheltenham.

Leitner, S. (2005): Conservative Familialism Revisited: The Case of Belgium, Acta Politica, 40(4), S. 419-439.

- (2003): Varieties of familialism: the caring function of the family in comparative perspective, European Societies, 5(4), pp. 353-75.

Lewis, J. (2001): The Decline of the Male Breadwinner Model: Implications for Work and Care, Social Politics, $8(2)$, S. $152-169$.

Marshall, T. H. (1964): Citizenship and social class, in: Marshall, T. H. (Hrsg.), Class, citizenship, and social development, Essays, Garden City, S. 65-122.

Merla, L. (2018): Belgium country note, in: Blum, S./Koslowski, A./Moss, P. (Hrsg.), International Review of Leave Policies and Related Research 2017. Abrufbar unter: http://www.leavenetwork.org/lp_and_r_reports/ (4.6.2018)

McKay, L./Mathieu, S./Doucet, A. (2016): Parental-leave rich and parental-leave poor: Inequality in Canadian labour market based policies, Journal of Industrial Relations, 58(4), S. 543-562. 
Orloff, Ann S. (1993): Gender and the social rights of citizenship: The comparative analysis of gender relations and welfare states, American Sociological Review, 58(3), S. 303-328.

Ray, R./Gornick, J. C./Schmitt, J. (2010): Who cares? Assessing generosity and gender equality in Parental Leave policy designs in 21 countries, Journal of European Social Policy, 19(5), S. 196-214.

Reimer, T./Erler, D./Blum, S. (2017): Germany country note, in: Blum, S./Koslowski, A./Moss, P. (Hrsg.), International Review on Leave Policies and Related Research 2017. Abrufbar unter: http://www.leavenetwork.org/lp_and_r_reports/ (4.6.2018)

Sainsbury, D. (Hrsg.) (1996): Gender, Equality and Welfare States, Cambridge: Cambridge University Press.

Saxonberg, S. (2013): From Defamilialization to Degenderization: Toward a New Welfare Typology, Social Policy and Administration, 47(1), S. 26-49.

Therborn, G. (1995): European Modernity and Beyond: The Trajectory of European Societies 1945-2000, London.

Vaughan-Whitehead D. (2012): Work Inequalities in the Crisis: Evidence from Europe, Cheltenham. 